\title{
Effect of Different Sowing Dates and Application of Selective Pesticides against Aphids (Lipaphis erysimi) Population in Mustard (Brassica juncea)
}

\section{Nagaraju Dharavath, Biswarup Mehera, Satyendra Nath, Sitanshu Sekhar Patra and Sandeep Rout*}

\section{School of Forestry \& Environment, Sam Higginbottom Institute of Agriculture Technology \& Sciences, Allahabad-211007 (Uttar Pradesh), India \\ *Corresponding author}

\section{A B S T R A C T}

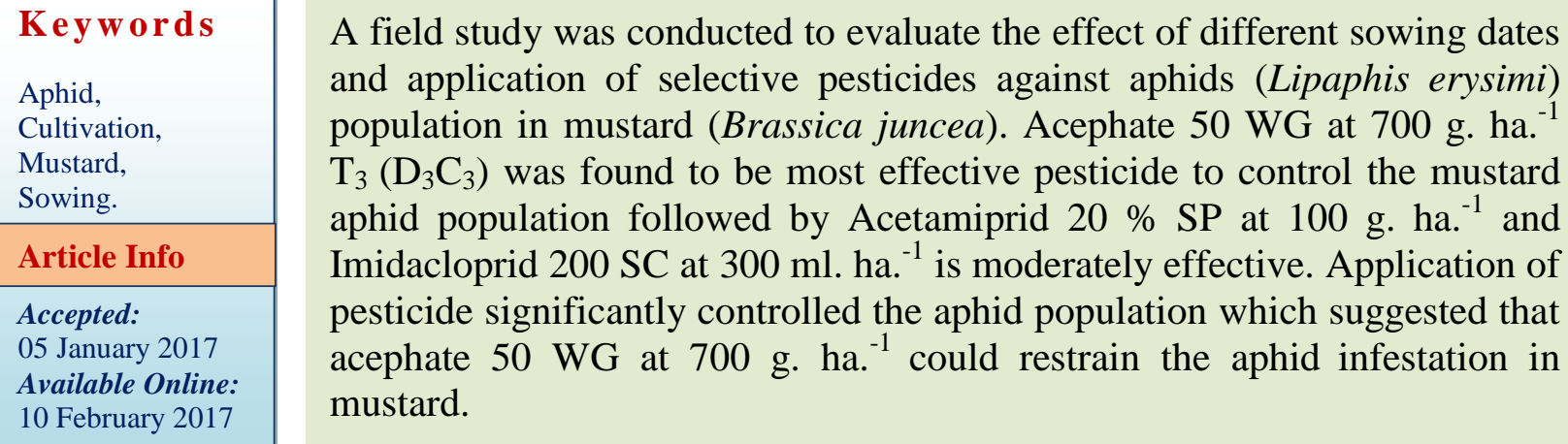

\section{Introduction}

Indian mustard (Brassica juncea) commonly known as raya, rai or laha is an important oilseed crop, among the Brassica group of oilseeds in India. It possesses a higher potential of production per unit area than other oleiferous members of the family Cruciferae. It is an annual plant or biennial herb. Rape seed and mustard are the major rabi oil seed crops of India. The origin of Indian mustard has been reported to be in China, it made its way into India through north eastern route.

The area, production and productivity of rape seed mustard in the world was $34.19 \mathrm{~m}$. ha, $63.09 \mathrm{~m}$. t, and $1850 \mathrm{Kg}$. ha ${ }^{-1}$ respectively, during the year 2012-2013. Globally India accounts for $19.29 \%$ and $11.127 \%$ of the total acreage and production (USDA, 2014).

In India, Brassica oil seeds are next to groundnuts in importance. Jammu and Kashmir, Punjab, Haryana, Uttar Pradesh, Rajasthan, Gujarat, Maharashtra, Odisha, West Bengal, Bihar and Assam are the important states growing mustard. Among these states, Rajasthan and Uttar Pradesh contribute the major share of mustard production in India (Gurujaran et al., 2008).

Oilseed crop does not require any special emphasis as these contribute a large share in 
the export of the commodities after meeting the international demands for industry and domestic condition. A small portion of the total production of the oilseed was retained by the cultivar for seed and domestic consumption, while the remaining is sold out. The oil extracted, beside being consumed in our diet, are used for non edible purposes such as the manufacture of vanaspati, soaps, paints varnishes, lubricants, greases, hair oils and tooth paste etc., the oil cakes obtained are used as cattle feed as well as manure. Mustard plays an important role in the oil seed economy of the country. It has 38 to $42 \%$ oil and 24\% protein (Maurya et al., 2012). Mustard is rich in minerals like Calcium, Manganese, Copper, Iron, Selenium, Zinc, Vitamin (A, B and C) and proteins.

The yield losses of mustard per year are one factor responsible for such loss is due to the ravage of insect pest attacking at various stages of the crop. Among them, Mustard aphids (L. erysimi) were the most devastating pest (Rouf and Kabir, 1997). The yield loss due to aphid infestation in mustard ranged from 87.16 to $98.16 \%$ (Anonymous, 1995). Abiotic factors seem to influence the aphid infestation due to large variation in the date of sowing, aphid infestation and its progress under favorable weather conditions. The average maximum and minimum relative humidity had a positive relationship with mean aphid infestation index. A minimum relative humidity of 30 to $35 \%$ and average maximum relative humidity of 85 to $88 \%$ were found to be the most congenial conditions for increase in aphid population (Narjary et al., 2013).

Amongst the various insect pests invading mustard crops, mustard aphid, Lipaphis erysimi is considered the most serious and responsible for a yield loss ranging from 35.4 to 96 percent depending upon seasons (Firake et al., 2013). The Aphid is the most serious pest of the mustard crop in North India causing yield loss up to $93 \%$ (Verma and Singh, 1987). The uses of insecticides have been recommended for the effective control of this pest (Prasad, 1997). Keeping in view the above facts the present study was conducted to evaluate the effect of different sowing dates and pesticides application against aphids.

\section{Materials and Methods}

The present investigation was carried out in the research field of the School of Forestry and Environment, Sam Higginbottom Institute of Agriculture, Technology and Sciences, Allahabad, during the period from October 2014 to March 2015, Rabi season. Allahabad is located in the South-East part of Uttar Pradesh India. The experimental site (Research field) is at an elevation of $98 \mathrm{MSL}$, at $25.57^{\circ} \mathrm{N}$ latitude, $81.51^{\circ} \mathrm{E}$ longitude. All the required materials and facilities necessary for the cultivation readily available in the department. This region has a subtropical climate with extreme of summer and winter. These locations receive the mean annual rainfall ranges from $500 \mathrm{~mm}$ to $1500 \mathrm{~mm}$. More than 70 per cent rains are received during S-W monsoon season 5 to 10 per cent rains are received in winter, $10-15$ per cent in summer and 5-10 per cent during post monsoon season. Normal rainy days exceed 40 per annum. Summer monsoonal rainfall comes in downpours while winter rainfall comes in light drizzles and is easily absorbed in soils. Temperatures vary greatly in these regions. May and June are the hottest and December and January is the coldest. Monthly mean temperature more than $25{ }^{\circ} \mathrm{C}$ prevails during 8-10 months of the year. During May and June maximum temperature rises above $40{ }^{\circ} \mathrm{C}$ and hot dry winds are common features. In January, the normal mean minimum temperature remains around 8 to $10^{\circ} \mathrm{C}$. Frost for one or two days may also 
occur during winter months (Patra et al., 2016). Pre-sowing operations i.e., the experimental field was prepared by cleaning and ploughing with the help of casual labours. The weeds were picked up in order to get clean and after that, it was leveled. For fertilizer application, the uniform dose of phosphorus and potash at $60,40 \mathrm{~kg} \mathrm{ha}^{-1}$ through DAP, MOP respectively and $40 \mathrm{~kg} \mathrm{~N}$ $\mathrm{ha}^{-1}$ through urea, were applied at the sowing time. The remaining amount of nitrogen (40 $\mathrm{kg} \mathrm{ha}^{-1}$ ) was applied at $41^{\text {st }}$ day after each date sowing in all plots. The Mustard variety used was Parsamani-8, make seeds as seeds were sown in rows at $30 \mathrm{~cm}$ apart at a depth of 2.5 $3 \mathrm{~cm}$ in the soil. The seed rate taken was 4.5 $\mathrm{kg} \mathrm{ha}^{-1}$. Post sowing operation i.e. Gap Filling were done after seed germination was observed on the $6^{\text {th }}, 7^{\text {th }}, 8^{\text {th }}$ day of $1^{\text {st }}, 2^{\text {nd }}, 3^{\text {rd }}$ sowing date respectively. Seeds were re-sown in the gaps where the previous seeds failed to germinate. Thinning was done at 25 days after every sowing when the crop has attained of 6 - 8 leaves. This operation was done for maintaining a proper plant to plant distance and standard plant population. Weeding was done by labours over on at $24^{\text {th }}$ November, $5^{\text {th }}$ and 22 December, 2014. This was done to maintain weed free environment during the initial crop growth stages.

Inter- cultivation was done by loosening the soil and earthing up was done while weeding for soil aeration for proper growth and development of plants and to protect from lodging. Irrigation was done on 45 days after the date of sowing for proper growth of plants. Second irrigation was done 75 days before pre-flowering of each sowing dates. The top dressing was done with the remaining half dose of nitrogen as urea at 47 days after sowing. Nitrogen was applied by furrow placement method at a distance of about 10 $\mathrm{cm}$ from the crop rows. The weather data were collected from the Meteorological observatory, SHIATS, Allahabad during the experimental period (Table 2). The experiment was carried out in a factorial design laid out as randomized block design with three replications (Gomez and Gomez, 1984). Each replication was divided into sixteen plots in the lengthwise direction, and the different treatments were allocated randomly in each replication. The experiment has two factors 1). Date of sowing- $\mathrm{D}_{1}$-First sowing date: $9^{\text {th }}$ October, $\mathrm{D}_{2}$ - Second sowing date: $19^{\text {th }}$ October, $\mathrm{D}_{3^{-}}$Third sowing date: $29^{\text {th }}$ October; 2). Insecticide- $\mathrm{C}_{1^{-}}$Control, $\mathrm{C}_{2 \text { - }}$ Imidacloprid $200 \mathrm{SC}$ at $(300 \mathrm{ml} / \mathrm{ha}),. \mathrm{C}_{3^{-}}$ Acetamiprid $20 \%$ SP (100 g / ha.), $\mathrm{C}_{4-}$ Acephate $50 \mathrm{WG}(700 \mathrm{~g} / \mathrm{ha}$.). The treatments details as follows (Table 1).

The observation on population dynamics of mustard aphids was recorded on the first day of every standard week between 09.00 to 13.30 hrs for untreated plots throughout the stand of a crop. When its appearance was first noticed, 5 plants were selected randomly and tagged to record aphids population in treated and untreated plots of each replication. The population was recorded from a $15 \mathrm{~cm}$ long top portion of central twig on a white paper sheet causing least possible disturbance as suggested by Singh and Singh (1982) and for treated plots observation recorded at $09.00 \mathrm{hrs}$ to $13: 00 \mathrm{hrs}$ as on $1^{\text {st }}$ Spraying one day before $1^{\text {st }}$ Spraying, $3^{\text {rd }}$ day after $1^{\text {st }}$ spraying, $7^{\text {th }}$ day after $1^{\text {st }}$ spraying and $15^{\text {th }}$ day after spraying. Similarly, the data were recorded after the second spraying in same manner.

\section{Results and Discussion}

The data on number of aphids per plant presented in table 3 reveal that, one day before spraying, the maximum number of aphids per plant was found in $\mathrm{T}_{4}$ (Second sowing date / Control) with 33.90 and minimum number of aphids per plant was observed in $\mathrm{T}_{9}$ (Third sowing / Imidacloprid $200 \mathrm{SC}$ ) with 16.20. It was found that there 
was non-significant effect by different sowing treatments. There was no significant effect of different sowing dates.

Three days after spraying it was found that there was significant effect by different sowing treatments. The maximum number of aphids per plant was found in $\mathrm{T}_{4}$ (Second sowing date / Control) with 1.64 and no aphids per plant was observed in $\mathrm{T}_{3}$ (First sowing / Acephate $50 \mathrm{WG}$ ), $\mathrm{T}_{7}$ (Second sowing / Acephate $50 \mathrm{WG}$ ), $\mathrm{T}_{9}$ (Third sowing / Imidacloprid $200 \mathrm{SC}$ ), $\mathrm{T}_{10}$ (Third sowing/ Acetamiprid $20 \%$ EC), $\mathrm{T}_{11}$ (Third sowing / Acephate $50 \mathrm{WG}$ ). Because of application of pesticides and also due to heavy rainfall the aphids are washed of 2 days after spraying. The present findings were in accordance of Bhatia and Sharma, (2014) who reported that among the insecticide Acephate $75 \mathrm{SP}$ at 350 $\mathrm{g}$ a.i./ ha. is most effective for destruction of aphids, followed by Acetamiprid $20 \mathrm{SP}$ at 10 $\mathrm{g}$ a.i./ ha after spraying of chemicals. Seven days after spraying it was found that there was significant effect by different sowing treatments. The maximum number of aphids per plant was found in $\mathrm{T}_{0}$ (First sowing date / Control) with 15.57 and a minimum number of aphids per plant was observed in $\mathrm{T}_{11}$ (Third sowing / Acephate 50 WG) with 1.00. On 15 days after spraying it was found that there was significant effect by different sowing dates. The maximum number of aphids per plant was found in $\mathrm{T}_{0}$ (First sowing date / Control) with 8.97 and minimum number of aphids per plant was observed in $\mathrm{T}_{11}$ (Third sowing / Acephate 50 WG) with 0.34 (Table 4). The decrease in temperature in January month also has significant negative effect on number of aphids without pesticides application (Nirmala et al., 2001).

Table.1 Treatment combinations of the experiment

\begin{tabular}{|c|c|l|l|}
\hline S. No. & Treatment & Treatment Combination & Code \\
\hline 1 & $\mathrm{~T}_{0}$ & First sowing date / Control & $\mathrm{D}_{1} \mathrm{C}_{1}$ \\
\hline 2. & $\mathrm{~T}_{1}$ & First sowing date / Imidacloprid 200 SC & $\mathrm{D}_{1} \mathrm{C}_{2}$ \\
\hline 3. & $\mathrm{~T}_{2}$ & First sowing date / Acetamiprid 20\% SP & $\mathrm{D}_{1} \mathrm{C}_{3}$ \\
\hline 4. & $\mathrm{~T}_{3}$ & First sowing date / Acephate 50 WG & $\mathrm{D}_{1} \mathrm{C}_{4}$ \\
\hline 5. & $\mathrm{~T}_{4}$ & Second sowing date / Control & $\mathrm{D}_{2} \mathrm{C}_{1}$ \\
\hline 6. & $\mathrm{~T}_{5}$ & Second sowing date / Imidacloprid 200 SC & $\mathrm{D}_{2} \mathrm{C}_{2}$ \\
\hline 7. & $\mathrm{~T}_{6}$ & Second sowing date / Acetamiprid 20\% SP & $\mathrm{D}_{2} \mathrm{C}_{3}$ \\
\hline 8. & $\mathrm{~T}_{7}$ & Second sowing date / Acephate 50 WG & $\mathrm{D}_{2} \mathrm{C}_{4}$ \\
\hline 9. & $\mathrm{~T}_{8}$ & Third sowing date / Control & $\mathrm{D}_{3} \mathrm{C}_{1}$ \\
\hline 10. & $\mathrm{~T}_{9}$ & Third sowing date / Imidacloprid 200 SC & $\mathrm{D}_{3} \mathrm{C}_{2}$ \\
\hline 11. & $\mathrm{~T}_{10}$ & Third sowing date / Acetamiprid 20\% SP & $\mathrm{D}_{3} \mathrm{C}_{3}$ \\
\hline 12. & $\mathrm{~T}_{11}$ & Third sowing date / Acephate 50 WG & $\mathrm{D}_{3} \mathrm{C}_{4}$ \\
\hline
\end{tabular}


Table.2 Mean weekly weather parameters during crop growing season (Rabi 2014-2015)

\begin{tabular}{|c|c|c|c|c|c|c|c|}
\hline \multirow[t]{2}{*}{ Standard week } & \multicolumn{2}{|c|}{ Temperature } & \multicolumn{2}{|c|}{$\begin{array}{l}\text { Relative } \\
\text { humidity }\end{array}$} & \multirow[t]{2}{*}{$\begin{array}{l}\text { Rainfall } \\
(\mathbf{m m})\end{array}$} & \multirow{2}{*}{$\begin{array}{l}\text { Sun shine } \\
\text { hours } \\
\text { (hrs) }\end{array}$} & \multirow{2}{*}{$\begin{array}{l}\text { Wind } \\
\text { speed } \\
(\mathbf{k m} / \mathbf{h r})\end{array}$} \\
\hline & $\mathbf{T}_{\max }$ & $\mathbf{T}_{\min }$ & 7 a.m & $\begin{array}{l}2 \\
\text { p.m }\end{array}$ & & & \\
\hline \multicolumn{8}{|l|}{ October } \\
\hline 41 & 30.63 & 25.82 & 84.14 & 49.42 & 0 & 7.6 & 1.07 \\
\hline 42 & 32.01 & 21.81 & 87 & 62.42 & 15.8 & 6.08 & 1.91 \\
\hline 43 & 33.24 & 20.14 & 87 & 53.28 & 0 & 8.42 & 0.69 \\
\hline 44 & 32.6 & 20.31 & 85.71 & 52.57 & 0 & 8.28 & 0.55 \\
\hline \multicolumn{8}{|l|}{ November } \\
\hline 45 & 33.17 & 19.57 & 86.28 & 45.71 & 0 & 8.18 & 0.81 \\
\hline 46 & 32.25 & 16.57 & 87 & 46.71 & 0 & 8.25 & 0.6 \\
\hline 47 & 29.71 & 12.02 & 85 & 45.28 & 0 & 8 & 0.66 \\
\hline 48 & 30.57 & 11.14 & 82.28 & 43.14 & 0 & 8.1 & 0.55 \\
\hline \multicolumn{8}{|l|}{ December } \\
\hline 49 & 30.54 & 12.37 & 82.85 & 44 & 0 & 8.38 & 0.6 \\
\hline 50 & 29.08 & 8.2 & 86 & 47.28 & 0 & 8.21 & 0.7 \\
\hline 51 & 24.68 & 9.45 & 89 & 60.42 & 1.2 & 5.08 & 1.28 \\
\hline 52 & 19.17 & 7.9 & 94.57 & 58.71 & 0 & 0.64 & 1.15 \\
\hline \multicolumn{8}{|l|}{ January } \\
\hline 1 & 17.62 & 10.85 & 93.42 & 64 & 4.71 & 0 & 1.31 \\
\hline 2 & 22.62 & 11.82 & 91 & 57.42 & 0 & 0 & 1.47 \\
\hline 3 & 15 & 7.7 & 93.71 & 72.14 & 0 & 0 & 1.34 \\
\hline 4 & 18.82 & 9.24 & 93.71 & 64.85 & 1.16 & 0 & 0.28 \\
\hline 5 & 19.65 & 13.04 & 91.14 & 63.14 & 1.76 & 1.2 & 4.57 \\
\hline \multicolumn{8}{|l|}{ February } \\
\hline 6 & 27.22 & 12.37 & 89.57 & 54.28 & 1.85 & 3.4 & 0.28 \\
\hline 7 & 26.48 & 11.31 & 89.14 & 49.57 & 1.44 & 4.54 & 0.45 \\
\hline 8 & 30.54 & 12.62 & 86.28 & 47.42 & 1.25 & 6.82 & 0 \\
\hline 9 & 30.81 & 14.65 & 87.28 & 50.71 & 1.92 & 6.86 & 0 \\
\hline \multicolumn{8}{|l|}{ March } \\
\hline 10 & 28.62 & 14.68 & 93.57 & 62 & 2.26 & 4.61 & 4.17 \\
\hline 11 & 31.6 & 15.8 & 85.42 & 45.14 & 1.25 & 7.74 & 0 \\
\hline
\end{tabular}


Table.3 Effect of different sowing dates and application of pesticides one before and three days after 1st spraying on number of aphids plant ${ }^{-1}$.

One day before

3 days after spraying

\begin{tabular}{|c|c|c|c|c|c|c|c|c|c|c|}
\hline \multirow[b]{2}{*}{$\begin{array}{l}\text { Date of sowing } \\
\text { (D) }\end{array}$} & \multicolumn{4}{|c|}{ Name of Pesticide (C) } & \multicolumn{5}{|c|}{ Name of Pesticide (C) } & \multirow[b]{2}{*}{$\begin{array}{l}\text { Mean } \\
\text { (D) }\end{array}$} \\
\hline & $\begin{array}{c}\text { Control } \\
\left(\mathrm{C}_{1}\right)\end{array}$ & $\begin{array}{l}\text { Imidacloprid } \\
200 \mathrm{SC}\left(\mathrm{C}_{2}\right)\end{array}$ & $\begin{array}{l}\text { Acetamiprid } \\
20 \% \mathrm{SP}\left(\mathrm{C}_{3}\right)\end{array}$ & $\begin{array}{c}\text { Acephate } \\
50 \mathrm{WG}\left(\mathrm{C}_{4}\right)\end{array}$ & $\begin{array}{l}\text { Mean } \\
\text { (D) }\end{array}$ & $\begin{array}{c}\text { Control } \\
\left(\mathrm{C}_{1}\right)\end{array}$ & $\begin{array}{l}\text { Imidacloprid } \\
200 \mathrm{SC}\left(\mathrm{C}_{2}\right)\end{array}$ & $\begin{array}{c}\text { Acetamiprid } \\
20 \% \mathrm{SP} \\
\left(\mathrm{C}_{3}\right)\end{array}$ & $\begin{array}{c}\text { Acephate } \\
50 \mathrm{WG} \\
\left(\mathrm{C}_{4}\right)\end{array}$ & \\
\hline $\begin{array}{l}1^{\text {st }} \text { date of sowing } \\
\left(D_{1}\right)\end{array}$ & 19.68 & 18.84 & 30.68 & 29.26 & 24.62 & 2 & 1.33 & 0.66 & 0 & 1 \\
\hline $\begin{array}{l}2^{\text {nd }} \text { date of sowing } \\
\left(D_{2}\right)\end{array}$ & 33.90 & 28.48 & 25.79 & 23.78 & 27.99 & 1.64 & 0.66 & 0.33 & 0 & 0.66 \\
\hline $\begin{array}{l}3^{\text {rd }} \text { date of sowing } \\
\left(\mathrm{D}_{3}\right)\end{array}$ & 22.69 & 16.20 & 16.81 & 16.95 & 18.16 & 1.33 & 0 & 0 & 0 & 0.33 \\
\hline \multirow[t]{2}{*}{ Mean $(C)$} & 25.42 & 21.17 & 24.43 & 23.33 & & 1.66 & 0.66 & 0.33 & $\mathbf{0}$ & \\
\hline & & F-test & S. Em. $( \pm)$ & C.D. at $5 \%$ & & & F-test & S. Em. $( \pm)$ & $\begin{array}{c}\text { C.D. at } \\
5 \%\end{array}$ & \\
\hline Date (D) & & NS & 3.781 & 8.09 & & & $\mathrm{~S}$ & 0.26 & 0.41 & \\
\hline Pesticide (C) & & NS & 4.384 & 9.341 & & & $\mathrm{~S}$ & 0.282 & 0.455 & \\
\hline Int. (D x C) & & NS & 7.681 & 16.18 & & & $\mathrm{~S}$ & 0.4 & 0.701 & \\
\hline
\end{tabular}


Table.4 Effect of different sowing dates and application of pesticides seven days and fifteen days after 1st spraying on number of aphids plant ${ }^{-1}$

7 days after $1^{\text {st }}$ spraying $\quad 15$ days after $1^{\text {st }}$ spraying

\begin{tabular}{|c|c|c|c|c|c|c|c|c|c|c|}
\hline \multirow[b]{2}{*}{$\begin{array}{l}\text { Date of sowing } \\
\text { (D) }\end{array}$} & \multicolumn{4}{|c|}{ Name of Pesticide (C) } & \multirow[b]{2}{*}{$\begin{array}{l}\text { Mean } \\
\text { (D) }\end{array}$} & \multicolumn{4}{|c|}{ Name of Pesticide (C) } & \multirow[b]{2}{*}{$\begin{array}{l}\text { Mean } \\
\text { (D) }\end{array}$} \\
\hline & $\begin{array}{l}\text { Control } \\
\left(\mathrm{C}_{1}\right)\end{array}$ & $\begin{array}{l}\text { Imidacloprid } \\
200 \mathrm{SC}\left(C_{2}\right)\end{array}$ & $\begin{array}{c}\text { Acetamiprid } \\
20 \% \mathrm{SP} \\
\left(\mathrm{C}_{3}\right)\end{array}$ & $\begin{array}{c}\text { Acephate } \\
50 \mathrm{WG} \\
\left(\mathrm{C}_{4}\right)\end{array}$ & & $\begin{array}{l}\text { Control } \\
\left(\mathrm{C}_{1}\right)\end{array}$ & $\begin{array}{l}\text { Imidacloprid } \\
200 \mathrm{SC}\left(\mathrm{C}_{2}\right)\end{array}$ & $\begin{array}{c}\text { Acetamiprid } \\
20 \% \mathrm{SP} \\
\left(\mathrm{C}_{3}\right)\end{array}$ & $\begin{array}{c}\text { Acephate } \\
50 \mathrm{WG} \\
\left(\mathrm{C}_{4}\right) \\
\end{array}$ & \\
\hline $\begin{array}{l}1^{\text {st }} \text { date of sowing } \\
\left(D_{1}\right)\end{array}$ & 15.57 & 3.64 & 2.34 & 2.00 & 5.89 & 8.97 & 2.64 & 1.00 & 0.34 & 3.24 \\
\hline $\begin{array}{l}2^{\text {nd }} \text { date of sowing } \\
\left(D_{2}\right)\end{array}$ & 10.31 & 2.64 & 2.00 & 1.64 & 4.15 & 7.63 & 2.00 & 1.64 & 1.33 & 3.15 \\
\hline $\begin{array}{l}3{ }^{\text {rd }} \text { date of sowing } \\
\left(\mathrm{D}_{3}\right)\end{array}$ & 4.51 & 1.34 & 1.36 & 1.00 & 2.05 & 3.86 & 2.00 & 1.66 & 0.34 & 1.97 \\
\hline \multirow[t]{2}{*}{$\operatorname{Mean}(\mathrm{C})$} & 10.13 & 2.54 & 1.90 & 1.55 & & 6.82 & 2.21 & 1.43 & 0.67 & \\
\hline & & F-test & S. Em. ( \pm$)$ & $\begin{array}{l}\text { C.D. at } \\
5 \%\end{array}$ & & & F-test & S. Em. ( $( \pm)$ & $\begin{array}{l}\text { C.D. at } \\
5 \%\end{array}$ & \\
\hline Date (D) & & $S$ & 0.756 & 1.439 & & & S & 0.614 & 1.145 & \\
\hline Pesticide (C) & & $\mathrm{S}$ & 0.854 & 1.643 & & & S & 0.691 & 1.303 & \\
\hline Int. ( $\mathrm{D} \times \mathrm{C})$ & & S & 1.392 & 2.758 & & & S & 1.108 & 2.17 & \\
\hline
\end{tabular}


Table.5 Effect of different sowing dates and application of pesticides one day before and three days after 2nd spraying on number of aphids plant ${ }^{-1}$

1 day before $2^{\text {nd }}$ spraying

\begin{tabular}{|c|c|c|c|c|c|c|c|c|c|c|}
\hline \multirow[b]{2}{*}{$\begin{array}{l}\text { Date of sowing } \\
\text { (D) }\end{array}$} & \multicolumn{4}{|c|}{ Name of Pesticide $(\mathrm{C})$} & \multirow[b]{2}{*}{$\begin{array}{c}\text { Mean } \\
\text { (D) }\end{array}$} & \multicolumn{4}{|c|}{ Name of Pesticide (C) } & \multirow[b]{2}{*}{$\begin{array}{c}\text { Mean } \\
\text { (D) }\end{array}$} \\
\hline & $\begin{array}{l}\text { Control } \\
\left(\mathrm{C}_{1}\right)\end{array}$ & $\begin{array}{l}\text { Imidacloprid } \\
200 \mathrm{SC}\left(\mathrm{C}_{2}\right)\end{array}$ & $\begin{array}{c}\text { Acetamiprid } \\
20 \% \mathrm{SP} \\
\left(\mathrm{C}_{3}\right)\end{array}$ & $\begin{array}{c}\text { Acephate } \\
50 \mathrm{WG} \\
\left(\mathrm{C}_{4}\right)\end{array}$ & & $\begin{array}{l}\text { Control } \\
\left(\mathrm{C}_{1}\right)\end{array}$ & $\begin{array}{l}\text { Imidacloprid } \\
200 \mathrm{SC}\left(\mathrm{C}_{2}\right)\end{array}$ & $\begin{array}{c}\text { Acetamiprid } \\
20 \% \mathrm{SP} \\
\left(\mathrm{C}_{3}\right)\end{array}$ & $\begin{array}{c}\text { Acephate } \\
50 \mathrm{WG} \\
\left(\mathrm{C}_{4}\right)\end{array}$ & \\
\hline $\begin{array}{l}1^{\text {st }} \text { date of } \\
\text { sowing }\left(D_{1}\right)\end{array}$ & 35.56 & 14.35 & 10.66 & 16.06 & 19.16 & 15.59 & 2.34 & 1.00 & 0.66 & 4.90 \\
\hline $\begin{array}{l}2^{\text {nd }} \text { date of } \\
\text { sowing }\left(D_{2}\right)\end{array}$ & 31.56 & 15.50 & 18.32 & 21.34 & 21.68 & 59.89 & 3.34 & 1.67 & 0.34 & 16.31 \\
\hline $\begin{array}{l}3^{\text {rd }} \text { date of } \\
\text { sowing }\left(D_{3}\right)\end{array}$ & 39.45 & 13.54 & 20.33 & 23.32 & 24.16 & 96.46 & 3.34 & 2.34 & 0.64 & 25.70 \\
\hline $\operatorname{Mean}(\mathrm{C})$ & 35.52 & 14.46 & 16.44 & 20.24 & & 57.31 & 3.01 & 1.67 & 0.55 & \\
\hline & & F-test & S. Em. $( \pm)$ & $\begin{array}{l}\text { C.D. at } \\
5 \%\end{array}$ & & & F-test & S. Em. ( \pm$)$ & $\begin{array}{l}\text { C.D. at } \\
5 \%\end{array}$ & \\
\hline Date (D) & & $\mathrm{S}$ & 1.734 & 3.725 & & & $\mathrm{~S}$ & 3.218 & 6.803 & \\
\hline Pesticide (C) & & $\mathrm{S}$ & 2.021 & 4.319 & & & $\mathrm{~S}$ & 3.734 & 7.874 & \\
\hline Int. $(\mathrm{D} \times \mathrm{C})$ & & NS & 3.588 & 7.569 & & & $\mathrm{~S}$ & 6.556 & 13.725 & \\
\hline
\end{tabular}


Table.6 Effect of different sowing dates and application of pesticides seven days and fifteen days after 2nd spraying on number of aphids plant ${ }^{-1}$

7 days after $2^{\text {nd }}$ spraying 15 days after $2^{\text {nd }}$ spraying

\begin{tabular}{|c|c|c|c|c|c|c|c|c|c|c|}
\hline \multirow[b]{2}{*}{$\begin{array}{l}\text { Date of sowing } \\
\text { (D) }\end{array}$} & \multicolumn{4}{|c|}{ Name of Pesticide (C) } & \multirow[b]{2}{*}{$\begin{array}{l}\text { Mean } \\
\text { (D) }\end{array}$} & \multicolumn{4}{|c|}{ Name of Pesticide (C) } & \multirow[b]{2}{*}{$\begin{array}{c}\text { Mean } \\
\text { (D) }\end{array}$} \\
\hline & $\begin{array}{c}\text { Control } \\
\left(\mathrm{C}_{1}\right)\end{array}$ & $\begin{array}{l}\text { Imidacloprid } \\
200 \mathrm{SC}\left(\mathrm{C}_{2}\right)\end{array}$ & $\begin{array}{c}\text { Acetamiprid } \\
20 \% \mathrm{SP} \\
\left(\mathrm{C}_{3}\right)\end{array}$ & $\begin{array}{c}\text { Acephate } \\
50 \mathrm{WG} \\
\left(\mathrm{C}_{4}\right)\end{array}$ & & $\begin{array}{l}\text { Control } \\
\left(\mathrm{C}_{1}\right)\end{array}$ & $\begin{array}{l}\text { Imidacloprid } \\
200 \mathrm{SC}\left(\mathrm{C}_{2}\right)\end{array}$ & $\begin{array}{c}\text { Acetamiprid } \\
20 \% \mathrm{SP} \\
\left(\mathrm{C}_{3}\right)\end{array}$ & $\begin{array}{c}\text { Acephate } \\
50 \mathrm{WG} \\
\left(\mathrm{C}_{4}\right)\end{array}$ & \\
\hline $\begin{array}{l}1^{\text {st }} \text { date of sowing } \\
\left(D_{1}\right)\end{array}$ & 3.34 & 0.00 & 0.00 & 0.00 & 0.84 & 0.00 & 0.00 & 0.00 & 0.00 & $\mathbf{0 . 0 0}$ \\
\hline $\begin{array}{l}2^{\text {nd }} \text { date of sowing } \\
\left(D_{2}\right)\end{array}$ & 31.66 & 2.67 & 2.34 & 1.66 & 9.58 & 0.00 & 0.00 & 0.00 & 0.00 & 0.00 \\
\hline \multirow{3}{*}{$\begin{array}{l}3^{\text {rd }} \text { date of sowing } \\
\left(D_{3}\right)\end{array}$} & 130.40 & 21.78 & 11.24 & 8.08 & 42.88 & 7.08 & 2.66 & 1.67 & 0.34 & 2.94 \\
\hline & 55.13 & 8.15 & 4.53 & 3.25 & & 2.36 & $\mathbf{0 . 8 9}$ & 0.56 & 0.11 & \\
\hline & & F-test & S. Em. $( \pm)$ & $\begin{array}{c}\text { C.D. at } \\
5 \%\end{array}$ & & & F-test & S. Em. $( \pm)$ & $\begin{array}{c}\text { C.D. at } \\
5 \%\end{array}$ & \\
\hline Date (D) & & S & 3.477 & 7.341 & & & S & 0.248 & 0.386 & \\
\hline Pesticide (C) & & S & 4.034 & 8.495 & & & $S$ & 0.268 & 0.428 & \\
\hline Int. $(\mathrm{D} \times \mathrm{C})$ & & S & 7.075 & 14.802 & & & S & 0.377 & 0.653 & \\
\hline
\end{tabular}


In the case of second spraying the data on number of aphids per plant presented in tables 5 and 6 revealed that: On one day before spraying, it was found that there was a significant effect in different date of sowing. The maximum number of aphids per plant was found in $\mathrm{T}_{8}$ (Third sowing date / Control) with 39.45 and a minimum number of aphids per plant was observed in $\mathrm{T}_{2}$ (First sowing date / Acetamiprid 20\% SP) with 10.66 numbers. Three days after spraying it was found that there was a significant difference within the treatments. The maximum number of aphids per plant was found in $\mathrm{T}_{4}$ (Second sowing date / Control) with 96.46 and a minimum number of aphids per plant was observed in $\mathrm{T}_{3}$ (Second date of sowing / Acephate $50 \mathrm{WG}$ ) with 0.34 . Khedkar et al. (2012) also reported that the application of Imidacloprid 17.8 SL (0.008\%), Acetamiprid 20 SP $(0.01 \%)$ and Acephate $(0.075 \%)$ was most effective for to control of the population of mustard aphids. In case of seven days after spraying it was observed that the maximum number of aphids per plant was found in $T_{8}$ (Third sowing date / Control) with 130.40 and no aphids per plant was observed in $\mathrm{T}_{1}$ (First sowing date / Imidacloprid $200 \mathrm{SC}$ ) $\mathrm{T}_{2}$ (First sowing date / Acetamiprid 20\% EC) $\mathrm{T}_{3}$ (First sowing date / Acephate $50 \mathrm{WG}$ ). This may be due to the gradual increase in temperature $\left(32^{0} \mathrm{C}\right)$ and the crop was forced to maturity further it reduced to zero number of aphids.

15 days after spraying found that there was significant effect by different sowing treatments. The maximum number of aphids per plant was found in $\mathrm{T}_{8}$ (Third sowing date / Control ) with 7.08 and no aphids per plant was observed in all treatments of first sowing and second sowing dates. Because of gradually increase in temperature $\left(32.6^{0} \mathrm{C}\right)$ and also crop is forced to maturity further it reduced to zero number of aphids. Hugar and Pratiba (2008) aslo found that the with the increase in temperature there is decrease in population of aphids. All the findings are more or less in agreement with the findings of the present study. Few variation were found which might be due to the variation of doses of insecticides and place (Maula et al. 2010).

It was concluded that all of the three sowing dates with the application of Acephate $50 \mathrm{WG}$ at $700 \mathrm{~g}$. ha. ${ }^{-1} \mathrm{~T}_{3}\left(\mathrm{D}_{3} \mathrm{C}_{3}\right)$ is most effective to control the mustard aphid population followed by Acetamiprid $20 \% \mathrm{SP}$ at $100 \mathrm{gm}$. ha. ${ }^{-1}$ and Imidacloprid $200 \mathrm{SC}$ at $300 \mathrm{ml}$. ha. ${ }^{-1}$ are moderately effective.

\section{References}

Anonymous. 1995. Oil Seed Development Strategies. National Oil seed Dev. Proj. Ministry of Food, Agric. \& Livest. Govt. of Pakistan. $120 \mathrm{Pp}$.

Bhatia, R. and Sharma, R.C. 2014. Efficacy of newer chemicals against mustard aphid. Biolife, 2(4):1165-1169.

Firake, D.M., Behere, G.T. and Thakur, A.N. S.2013.Eco-friendly management of mustard aphid, Lipaphis erysimi: A Most Destructive Pest of Brassicaceous crops. J. Popular Kheti, 1(3).

Gomez, K.A. and Gomez, A.A. 1984. Statistical procedures for Agricultural Res. 2nd edn. John Wiley and Sons, New York. 680 Pp.

Gurujaran, B., Balasubramanian, R. and Swaminathan, V. 2008. Recent strategies on crop production. Kalyani Publishers, New Delhi.159-160p

Hugar, G. and Pratiba, K. 2008.Seasonal incidence of important insect pests of mustard and there natural enemies in relation to weather parameters in Allahabad region. J. Entomol. Res., 32(2): 109-112.

Khedkar, A.A., Bharpoda, T.M., Patel, M.G. and Patel, C.K. 2012. Efficacy of different chemical against mustard aphid, Lipaphis erysimi (kaltenbach) 
infesting mustard. AGRES - an Int. $e$ J., 1(1): 53-64.

Maula, A., Shah, M., Siddquie, N., Mamun, M. and Begum, M. 2010. Effectiveness of three Insecticides against Mustard Aphid and Predator under field condition. Bangladesh J. Agri. Res., 35(1): 179-187.

Maurya, N., Singh, A.K. and Singh, S.K. 2012. Analysis of combining ability in Indian mustard (Brassica juncea). Indian J. Plant Sci., 1(2-3):116-123.

Narjary, B., Adak, T., Meena, M.D. and Chakravarty, N.V.K. 2013. Population dynamics of mustard aphid in relation to humid thermal ratio and growing degree days. J. Agri. Physics, 13(1): 39-47

Nirmala, D., Indra, D., Desh, R., Devi, N., Dogra, I. and Raj, D. 2001. Comparative field efficacy of insecticides against mustard aphid ( $L$. erysimi) on rapeseed crop in mid hill zone of Himachal Pradesh. J. Entomol. Res., 25(2): 93-96.

Patra, S.S., Mehera, B., Rout, S., Tomar, S.S., Singh, M. and Kumar, R. 2016. Effect of hydropriming and different sowing dates on growth and yield attributes of Wheat (Triticum aestivum L.). J. Appl. Natural Sci., 8(2): 971-980.

Prasad, S.K. 1997. Efficacy of some neem products vis- a- vis oxydemeton methyl against Lipaphis erysimi (kalt.) on rapeseed crop under field condition. Indian J. Entomol., 59(2): 147-150.

Rouf, F.M.A. and Kabir. K.H. 1997. Economic efficacy of some insecticides for the control of mustard aphid, $L$. erysimi Kalt. Bangladesh J. Entomol., 7(1\&2): $1-7$.

Singh, H.S. and Singh, R. 1982. Effect of cropping system on the incidence of mustard aphids (Lipaphis erysimi) (Kalt.) and natural enemies under the agro climatic condition of Eastern Uttar Pradesh. Shaspa, 7(1): 41-47.

USDA. 2014. World market and trade.

Verma, S.N. and Singh, O.P. 1987. Estimation of avoidable losses to mustard by the aphid, Lipaphis erysimi in Madhya Pradesh. Indian J. Plant Protection, 15(1): 87-89.

\section{How to cite this article:}

Nagaraju Dharavath, Biswarup Mehera, Satyendra Nath, Sitanshu Sekhar Patra and Sandeep Rout. 2017. Effect of Different Sowing Dates and Application of Selective Pesticides against Aphids (Lipaphis erysimi) Population in Mustard (Brassica juncea). Int.J.Curr.Microbiol.App.Sci. 6(2): 56-66. doi: http://dx.doi.org/10.20546/ijcmas.2017.602.008 